\title{
Sensitivity of the surface orographic gravity wave drag to vertical wind shear over Antarctica
}

Article

Accepted Version

Turner, H. V., Teixeira, M. A. C., Methven, J. and Vosper, S. B. (2019) Sensitivity of the surface orographic gravity wave drag to vertical wind shear over Antarctica. Quarterly Journal of the Royal Meteorological Society, 145 (718). pp. 164-178. ISSN 1477-870X doi: https://doi.org/10.1002/qj.3416 Available at https://centaur.reading.ac.uk/79776/

It is advisable to refer to the publisher's version if you intend to cite from the work. See Guidance on citing.

To link to this article DOI: http://dx.doi.org/10.1002/qj.3416

Publisher: Royal Meteorological Society

All outputs in CentAUR are protected by Intellectual Property Rights law, including copyright law. Copyright and IPR is retained by the creators or other copyright holders. Terms and conditions for use of this material are defined in the End User Agreement.

www.reading.ac.uk/centaur 
Central Archive at the University of Reading

Reading's research outputs online 
variation of the velocity with height, and directional wind shear with a velocity that turns with height at a constant rate whilst keeping its magnitude constant. In the case of unidirectional shear, the drag in the direction aligned with the flow was found to decrease with decreasing Ri (i.e. increasing shear), whereas (not surprisingly) no drag occurred in the perpendicular direction (Teixeira et al. 2004). For the directional shear with a linear change of the velocity with height, the drag in the direction aligned with the flow at the surface decreased with increasing shear at the same rate as in the previous case, whilst the drag in the perpendicular direction decreased $1 / 3$ more slowly, leading to a misalignment of the drag force with the surface wind vector (Teixeira et al. 2004). Note that in both of the preceding wind profiles, the wind speed is unbounded. In the final case of the wind that turns with height with constant magnitude, the drag was found instead to increase with increasing shear (decreasing Ri), due to terms involving the curvature of the wind profile (Teixeira et al. 2004). The theoretical model suggests that a similar behaviour should extend to most wind profiles with directional shear (even non-idealized ones), because the wind velocity components and their second derivatives with respect to height tend to have opposite signs, so that the wind speed remains bounded (Miranda et al. 2009). It is important to note that shear effects on the surface drag were only captured in these calculations because a 2 nd-order WKB approximation was used, which was seen to be fairly accurate for values of Ri down to $O(1)$. All predictions from the model were shown to be in good agreement with data from numerical simulations of the inviscid, nonlinear, non-hydrostatic equations of motion run for low-amplitude mountains (Teixeira et al. 2004).

Teixeira and Miranda (2006) extended this work to test drag equations derived for flow over mountains with an elliptical horizontal cross-section, which are the approximation to real orography assumed in some prominent drag parametrizations (Lott and Miller 1997; Zadra et al. 2003). Otherwise, the model had a similar basis to that used by Teixeira et al. (2004). This model can also be viewed as an extension of the linear hydrostatic calculations developed by Phillips (1984) that allows the wind to vary in the vertical. Tests were performed for both a wind profile with a linear variation, and one in which the wind turns with height keeping a constant magnitude. The results indicated essentially similar changes in the drag to those found by Teixeira al. (2004) (with some differences in detail due to the orography geometry), thereby showing that such effects are important for this more general orographic profile (Teixeira and Miranda 2006). One these differences is that the drag corrections tend to be larger when the shear vector is directed across an elongated mountain rather than along it, which is consistent with the larger magnitude of the drag corrections found for a similar model developed for 2D flow (Teixeira and Miranda 2004). The predictions from the WKB model of Teixeira and Miranda (2006) were shown to be good for the first wind profile by comparison with numerical simulations and slightly less satisfactory for the second. However in all cases the model, as that of Teixeira et al. (2004), produced qualitatively accurate predictions and was of course asymptotically correct in the limit of high Ri.

The theoretical model developed by Teixeira and Miranda (2006) was employed by Miranda et al. (2009) to compute global fields of GWD using ERA-40 meteorological data and elliptical mountains with various aspect ratios, orientations, widths and heights, adopted in the ECMWF's GCM to represent the Earth's orography within each model grid box. This yielded a first evaluation of the formulae including a correction for shear under fairly realistic conditions. The impact of shear varied between regions, with the strongest relative impact being felt over eastern Africa and Article is protected by forpuright. Alt rights reserved the mean
GWD stress by a significant amount (Miranda et al. 2009). This suggested that shear may have a substantial impact on the drag at the global scale. Regionally, the GWD stress found by Miranda et al. (2009) over Antarctica was found to be enhanced by up to $50 \%$, and the impact of shear on GWD was seen to be important throughout the year in this region, but reaching a peak during June, July and August (JJA, i.e. Southern Hemisphere winter) (Miranda et al. 2009). Thus it was shown that the shear correction applied to the drag slightly reduced the known bias existing in the GWD torque, as diagnosed from the resolved flow and the current version of the drag parametrization (Miranda et al. 2009).

Although the study of Miranda et al. (2009) provided a starting point for investigating the impact of shear on GWD, many uncertainties concerning their results remain. Since the corrections to the drag due to shear are expressed at the surface in the inviscid model used to derive them, it is unclear what height to select from real atmospheric data, where a boundary layer affects the flow in ways not originally accounted for. Miranda et al. (2009) adopted the same height as used currently to estimate the wind velocity and static stability in the ECMWF GWD parametrization, but other choices are possible. In principle the optimal height is that which contributes most to the surface pressure perturbation associated with the mountain waves that cause the drag. However, determining that height objectively is non-trivial. This study presents sensitivity tests that intend to start to address this and other questions that remain open, namely:

- How sensitive are the drag corrections due to shear to the level in the atmosphere at which they are evaluated?

- How does the orography anisotropy affect these drag corrections?

- Which terms in the drag corrections are dominant, and how is their variability characterized?

- What fraction of the time does directional wind shear dominate the drag?

Miranda et al. (2009) used a height based on the standard deviation of the model subgrid-scale orography elevation within each grid box to evaluate the vertical derivatives associated with shear effects. In the present study, we will compare calculations using the same nominal height with calculations using another equally reasonable height based on the first reanalysis levels above the top of the diagnosed boundary layer. This latter height is chosen in order to avoid effects of boundary layer turbulence (which should be of minor importance to the dynamics of the mountain waves, and the drag associated with them) and yet be as near as possible to the surface.

This paper also aims to determine geographic areas with a large incidence of low values of $\mathrm{Ri}($ say, $\mathrm{Ri}<3$ ) at either height, and the seasonality of these patterns (since the Richardson number can be considered a measure of the importance of shear effects). Additionally, an examination is made of spatial patterns and temporal variability of the terms upon which the corrected drag depends, and of their magnitudes relative to each other. These terms depend not only on the first derivatives of the wind velocity, but also on the second derivatives (i.e. curvature terms), which are associated primarily with wind rotation with height (i.e. directional shear), and generally cause GWD enhancement (as explained above). These results are considered in the light of their potential impacts upon the drag.

Situations with $\mathrm{Ri}<0.25$ are effectively irrelevant for these calculations (and they do not occur in the main region we focus on anyway - see below), because they correspond to dynamic or even static instability, for which the gravity wave structure would be disrupted by turbulence (with unforeseen consequences). So in the cases where such values of Ri exist, the drag corrections would be set to zero in an operational parametrization context. It 
should also be noted that the $\mathrm{Ri}$ we consider in our calculations is the local gradient Richardson number at a height considered representative for the impact of shear on gravity waves, whereas the top of the boundary layer is defined in reanalysis based on the bulk Richardson number, an independent parameter.

Section 2 of this paper deals with the methodology used for the data processing. In section 3 the results pertaining to the dependence of the drag upon $\mathrm{Ri}$ are presented and discussed, as well as those related to analysis of the other terms on which the drag depends. Section 4 presents the main conclusions of this study.

\section{(1)}

\section{Methodology}

All calculations involving atmospheric parameters are carried out using the ECMWF ERA-Interim reanalysis dataset. The variables required for these calculations are stored on a grid with a maximum horizontal spacing of $78.2 \mathrm{~km}$ (corresponding to T255 spectral resolution) and 37 pressure levels - from the surface up to $1 \mathrm{hPa}$, at $00,06,12$ and 18 UTC each day from 1979 to 2015. The boundary layer height, which is given as the top of the turbulent mixed layer as diagnosed based on the bulk Richardson number (Sodemann et al. 2008), is stored separately at 00 and 12 UTC over the same time period. Pressure levels are used for convenience, since they have enough vertical resolution for the present purpose $(25 \mathrm{hPa}$, or approximately $250 \mathrm{~m}$, below 750 $\mathrm{hPa})$. In the interest of accuracy, only data at 00 and 12 UTC are used for all variables, so that a value of the boundary layer height, which is necessary for the calculations, is always available. Statistical parameters of the subgrid-scale orography within each model grid box (anisotropy, mean orientation angle, height, slope, etc.) are also available from the same dataset. In particular, the standard deviation of the orography elevation (a key parameter of - the orographic drag parametrization scheme) is available at the same spatial resolution as the boundary layer height.

As we are interested in the effects of shear upon GWD, we adopt two possible heights at which to evaluate these effects: 1 . immediately above the top of the atmospheric boundary layer (henceforth BLH), in order to avoid the effects of turbulence and mixing that may exist within that layer; 2. similar to the height currently used to evaluate $N$ and $(U, V)$ (the Brunt-Väisälä frequency and the mean incoming wind vector, respectively) in he ECMWF orographic GWD parametrization (henceforth SDH) (i.e. nominally the same height as selected by Miranda et al. 2009). In the case of the BLH, the mixing within the atmospheric boundary layer that we wish to avoid always leads to low values of Ri, which are not relevant to the (inviscid) aspects of orographic gravity waves we wish to focus on here. Note that by definition the BLH has a seasonal (and even diurnal) dependence, which does not exist in the SDH.

The procedure to estimate the impact of shear on the drag is as follows. First of all the Richardson number, which is a measure of/ shear intensity, is evaluated globally. This is defined as the gradient Richardson number (shown by Teixeira et al. (2004) and Teixeira and Miranda (2006) to be a key parameter on which the drag depends)

$$
R i=\frac{N^{2}}{\left(\frac{d U}{d z}\right)^{2}+\left(\frac{d V}{d z}\right)^{2}} .
$$

evaluated at a convenient level. To compute $\mathrm{Ri}$ at the BLH, introduced above, the lowest of the 3 pressure levels used to evaluate the derivatives necessary to calculate $\mathrm{Ri}$ is defined as the first level above the boundary layer height, with the intermediate and upper level defined as the consecutive levels immediately above. For the SDH, the first pressure level above a distance above the surface article is protacted by copsriaht. All tights reserved. deviation of the subgrid-scale terrain elevation within each grid box is defined as the first of three consecutive levels over which the same derivatives are evaluated.

The calculation is performed using centred finite differences (between the lowest and highest levels) for the vertical derivatives of $U$ and $V$, and of the potential temperature that are needed to compute $N^{2}$. Other quantities are approximated by their values at the intermediate level. Note that the SDH used here (like other subgrid-scale orography parameters) is necessarily different (in value, although not in definition) from that considered in the original study of Miranda et al. (2009), as we use the ERAInterim dataset, whereas those authors used ERA-40 data, which are provided at lower resolutions.

A similar method is employed in the calculation of the terms contained in the drag corrections due to shear formulated by Teixeira and Miranda (2006), which are in general not expressible directly in terms of the Richardson number. The terms concerned come from the equations for the drag used by Miranda et al. (2009), which may be written as follows:

$$
\begin{aligned}
D_{x}= & D_{0 x}\left[1-\frac{1}{8}\left\{\frac{\alpha}{N^{2}}\left(U^{\prime 2}+2 U U^{\prime \prime}\right)\right.\right. \\
& \left.\left.+\frac{1-\alpha}{N^{2}}\left(V^{\prime 2}+4 V V^{\prime \prime}\right)\right\}\right] \\
& -\frac{1}{4} D_{0 y} \frac{1-\alpha}{N^{2}} \frac{B}{C}\left(U^{\prime} V^{\prime}+V U^{\prime \prime}\right), \\
D_{y}= & D_{0 y}\left[1-\frac{1}{8}\left\{\frac{\beta}{N^{2}}\left(V^{\prime 2}+2 V V^{\prime \prime}\right)\right.\right. \\
& \left.\left.+\frac{1-\beta}{N^{2}}\left(U^{\prime 2}+4 U U^{\prime \prime}\right)\right\}\right] \\
& -\frac{1}{4} D_{0 x} \frac{1-\beta}{N^{2}} \frac{C}{B}\left(U^{\prime} V^{\prime}+U V^{\prime \prime}\right) .
\end{aligned}
$$

Here the primes indicate derivatives with respect to height $z$. Note that these terms involve not only first but also second wind derivatives. The corresponding finite-difference approximations make explicit use of all three discrete levels mentioned above. $D_{0 x}$ and $D_{0 y}$ are the values of the drag components in the absence of shear along the minor and major axes of the ellipse defining the mountain. $B, C, \alpha$ and $\beta$ are coefficients related to the horizontal aspect ratio of the mountain, $\gamma$, which is a parameter available directly from the ERA-Interim dataset for all points around the globe. These coefficients are defined as (Teixeira and Miranda 2006)

$$
\begin{aligned}
& B(\gamma)=\int_{0}^{\frac{\pi}{2}} \frac{\cos \theta^{2}}{\left(\cos \theta^{2}+\gamma^{2} \sin \theta^{2}\right)^{\frac{1}{2}}} d \theta, \\
& C(\gamma)=\gamma^{2} \int_{0}^{\frac{\pi}{2}} \frac{\sin \theta^{2}}{\left(\cos \theta^{2}+\gamma^{2} \sin \theta^{2}\right)^{\frac{1}{2}}} d \theta, \\
& \alpha(\gamma)=\frac{1}{1-\gamma^{2}}\left(1-\frac{C(\gamma)}{B(\gamma)}\right), \\
& \beta(\gamma)=\frac{\gamma^{2}}{1-\gamma^{2}}\left(\frac{B(\gamma)}{C(\gamma)}-1\right) .
\end{aligned}
$$

In order to test the effect of the orography anisotropy, we also consider the simpler case of an axisymmetric mountain, thereby avoiding the need to calculate the functions (4)-(7). The coefficients related to the aspect ratio of the mountain, in this case $\gamma=1$, then take the following constant values: $B=C=\frac{\pi}{4}$ and $\alpha=\beta=\frac{3}{4}$ (Teixeira and Miranda 2006) which, from (2)-(3), 
yields (Teixeira et al. 2004)

$$
\begin{aligned}
\frac{D_{x}}{D_{0}}= & {\left[1-\frac{1}{32 N^{2}}\left\{3\left(U^{\prime 2}+2 U U^{\prime \prime}\right)+V^{\prime 2}+4 V V^{\prime \prime}\right\}\right] } \\
& \times \cos \psi-\frac{1}{16 N^{2}}\left(U^{\prime} V^{\prime}+V U^{\prime \prime}\right) \sin \psi, \\
\frac{D_{y}}{D_{0}}= & {\left[1-\frac{1}{32 N^{2}}\left\{3\left(V^{\prime 2}+2 V V^{\prime \prime}\right)+U^{\prime 2}+4 U U^{\prime \prime}\right\}\right] } \\
& \times \sin \psi-\frac{1}{16 N^{2}}\left(U^{\prime} V^{\prime}+U V^{\prime \prime}\right) \cos \psi,
\end{aligned}
$$

where $D_{0}=\left(D_{0 x}^{2}+D_{0 y}^{2}\right)^{1 / 2}$ is the magnitude of the drag in the absence of shear and $\psi$ is the angle of the near-surface wind such that $\tan \psi=\frac{V}{U}=\frac{D_{0 y}}{D_{0 x}}$.

To evaluate the temporal variability of shear effects, the fraction of time at which the inverse of $\mathrm{Ri}$ is above a certain threshold

value is also calculated in a specified region. For this purpose, the value of the quantity being analysed at each grid-point for both times considered ( 00 and 12 UTC) is compared to the prescribed threshold. If the value is above the threshold, 1 is added to a count for the corresponding grid-point. The count is then divided by the number of total counts (i.e. 2 per day) over the given period. This method is also employed to compute the fraction of times at which second derivatives of the wind velocity dominate the drag corrections (as given by (2) and (3)).

\section{Results}

\subsection{A climatology of $R i$}

Four seasons are defined, corresponding to the months of December, January and February (DJF), March, April and May (MAM), June, July and August (JJA), and September, October and November (SON). Unless otherwise explicitly stated, we will focus in what follows on DJF, corresponding to Southern Hemisphere (SH) summer, and JJA, corresponding to SH winter, as these are typically the extreme seasons in terms of atmospheric behaviour. This turns out also to be the case for the drag, with few exceptions. Seasonal averages for DJF and JJA of the inyerse of $\mathrm{Ri}$ for the decade 2006-2015 (figure 1) reveal various regions where $\mathrm{Ri}^{-1}=O(1)$, suggesting potential impact of shear on GWD (since for simple wind profiles the corrections to the drag due to shear are inversely proportional to $\mathrm{Ri}$; cf. Teixeira et al. 2004; Teixeira and Miranda 2006). Figure 1 presents global plots of $\mathrm{Ri}^{-1}$ evaluated at the BLH (as defined above). Figure 2 shows the same, but focusing specifically on Antarctica and including also results evaluated at the SDH.

The results indicate that high shear occurs in various regions over the continents, particularly over the edge of Antarctica throughout the year, peaking in intensity during JJA, in agreement with Miranda et al. (2009), and weakening somewhat in DJF. Maximum values of $\mathrm{Ri}^{-1}$ in this region range between over 0.3 in DJF to over 0.5 in JJA for calculations performed using the BLH as the reference height (see figures 1 and 2(a),(b)), and exceeding 0.7 during all seasons (although such values are more widespread in JJA) for calculations using the SDH as the reference height (see figure 2(c),(d)). This suggests that the impact on drag could be substantial according to the theory of Teixeira et al. (2004) and Teixeira and Miranda (2006). Note that there are also high (in fact even higher) values of $\mathrm{Ri}^{-1}$ over the continents in various temperate and tropical regions in figure 1 (where only results calculated at the BLH are shown), e.g. the Sahara desert (especially in JJA), Europe and the US (especially in DJF), the Himalayas (in DJF), Australia, the southern half of South America, and East Asia. Some of these values (for example, those over the Sahara desert, Eurasia and North America in JJA), which are sometim article is protected by coporight All rights reserved
The drag diagnostic we propose (based on (2)-(3), where $N^{2}$ appears in denominator) is clearly not robust in such conditions, i.e., a parametrization based on it without any provision for the avoidance of dynamical or static instability, would behave erratically, as in the latter case $N^{2}$ oscillates around zero. So the regions where this happens will be disregarded.

The behaviour of $\mathrm{Ri}^{-1}$ over Antarctica (see figure 2) seems qualitatively more robust than elsewhere in the results of figure 1. This is due to the atmosphere being statically stable in this region, less dependent on seasonal cycle (and diurnal cycle - not shown), and therefore better suited to our sensitivity tests. As the Antarctic region exhibits high shear throughout the year (as shown by Miranda et al. (2009) and confirmed here), this is the most relevant area for investigation. Given the extremely cold climate of Antarctica, the boundary layer is there very shallow (see below), and so expected to affect the drag less, giving further relevance to the inviscid drag estimates presented here. Additionally, the drag over Antarctica has regional importance because it influences the dynamics of the polar vortex (Watanabe et al. 2006). We will, therefore, concentrate on this region for the remainder of this study.

Figure 3(a)-(c) shows histograms of the BLH over Antarctica in JJA ( $\mathrm{SH}$ winter), of the $\mathrm{SDH}$, and also a histogram of the difference between these two quantities. It can be seen that the BLH is typically very low, peaking at values between $10 \mathrm{~m}$ and $15 \mathrm{~m}$, presumably because of the very strong cooling to which the surface is subjected. The SDH can be even lower, peaking at values between $5 \mathrm{~m}$ and $10 \mathrm{~m}$, presumably because of contributions from the very flat interior of Antarctica. Curiously, the SDH has a larger frequency at higher values, of $150 \mathrm{~m}-200 \mathrm{~m}$, than the BLH, probably corresponding to the edge of Antarctica, where the orography variability is much more pronounced. Figure 3(d) shows a histogram of the BLH in DJF ( $\mathrm{SH}$ summer). Clearly, the BLH is much higher, and has much more variability (shown by the flatter distribution) during this season, therefore it must exceed the SDH in an even more pronounced way than in JJA.

From figures 1 and 2, in Antarctica the values of $\mathrm{Ri}^{-1}$ estimated using the BLH are systematically lower than those using the SDH. As seen above, the BLH typically takes larger values than the $\mathrm{SDH}$, which means that the SDH is usually within the atmospheric boundary layer. This is further corroborated by the histogram of the difference between BLH and SDH (figure 3(c)), which shows that this quantity is predominantly positive, although negative values do occur. Since shear tends, on average, to be concentrated near the surface and decrease with height, this explains why $\mathrm{Ri}^{-1}$ is typically higher when the SDH is used than when the BLH is used. Additionally, by a similar argument, figure 3(d) justifies why $\mathrm{Ri}^{-1}$ is lower in DJF than in JJA when the BLH is used: the BLH exceeds the SDH even more in that case. Especially for the BLH, the values of $\mathrm{Ri}^{-1}$ obtained from these temporal averages suggest levels of shear that would have only a modest impact upon the drag. However, they may underestimate the actual importance of shear over shorter time scales.

Figure 4 shows histograms of the values of Ri over Antarctica for $\mathrm{Ri}^{-1}$ averaged over different time periods (1 day, 1 month, 1 year, and the full period 2006-2015), using the BLH as the reference height. Because of the substantial variability that exists on a daily time scale, for the 1-day case histograms were averaged over all days of July 2015. The 1-month histogram is for July 2015, and the histogram for 1 year refers to the period of December 2014 to November 2015 (because this allows it to contain complete seasons, as defined above). These histograms indicate a higher incidence of low Ri values (between 1 and 2) when the averaging period is reduced, which is consistent with the hypothesis proposed above. The histograms peak between $\mathrm{Ri}=3$ and $\mathrm{Ri}=4$ over a day, between $\mathrm{Ri}=4$ and $\mathrm{Ri}=5$ over a month, and 
variability of $\mathrm{Ri}$ is due to the variation of the value of the BLH, and another (dominant) part is due to the variation of the wind shear at the BLH. In equivalent histograms calculated at the SDH (not shown), only the second part of the variability contributes.

To further explore this short timescale variability we employ the technique described in the preceding section to compute the fraction of the time at which $\mathrm{Ri}^{-1}$ is above a given threshold. Figure 5 shows the fraction of the time at which $\mathrm{Ri}^{-1}>1 / 3$ (i.e. $\mathrm{Ri}<3$ ) over Antarctica for each season during the decade 20062015 , for calculations done at both reference heights introduced previously. Again we see a high incidence of low values of Ri over the edge of Antarctica year-round, with a few spots where fraction of the time with $\mathrm{Ri}<3$ is above 0.8 , and maximum intensity during JJA, which is consistent with Miranda et al. (2009). Note that while the seasonal maximum values of the fractional occurrence of $\mathrm{Ri}<3$ do not differ very markedly when using the BLH or the SDH as reference heights, the seasonal cycle s substantially more pronounced when using the BLH, which is natural because the BLH varies seasonally whereas the SDH does not.

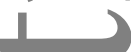

3.2. Effect of orography anisotropy on shear corrections to the drag

The drag corrections due to shear are calculated next using the formulae for elliptical mountains (2)-(3), which give the most accurate representation of anisotropic orography (as represented in a number of parametrizations), and compared with the results obtained assuming axisymmetric orography (8)-(9). By considering both of these orographic profiles we are able to build up a more complete picture of the factors that lead to drag enhancement (or reduction) due to shear, including its interaction with the orography anisotropy.

\subsubsection{Axisymmetric mountains}

As explained in section 2, approximating the orography as axisymmetric constitutes a considerable simplification of the drag corrections due to shear, which can be calculated without resorting to orographic parameters.

Figure 6 presents the correction to the drag magnitude due to shear for axisymmetric orography, defined based on expressions 8)-(9), as $D / D_{0}=\left(D_{x}^{2}+D_{y}^{2}\right)^{1 / 2} /\left(D_{0 x}^{2}+D_{0 y}^{2}\right)^{1 / 2}$. We can see enhancement of the drag along the edge of Antarctica both in DJF and JJA, but with maximum intensity during JJA for both reference heights. Actually, the drag enhancement attains a maximum during SON when the SDH is used, but the difference relative to JJA is small. In this case the seasonal cycle is not very well defined, partly because the SDH is fixed, and fairly strong amplifications are present throughout the year. In figure 6, the drag intensification with respect to that in the absence of shear suggests that directional shear dominates the shear correction. The terms with first derivatives in (2)-(3) or (8)-(9) are always necessarily negative - except that involving $U^{\prime} V^{\prime}$ - so almost only terms with second derivatives may enhance the drag. The possibility that it is the second derivatives that are mainly responsible for the drag enhancement seen in figure 6 is confirmed by the fact that figure 5(a),(b) and 5(c),(d) (or 2(a),(b) and 2(c),(d)) appear to differ less than figure 6(a),(b) and 6(c),(d). The former show statistics based on $\mathrm{Ri}$, which do not involve any second derivatives, whereas the latter show a quantity (the drag enhancement) that is affected by such derivatives. The magnitude of the enhancement seen when the $\mathrm{SDH}$ is used as a reference height (figure $6(\mathrm{c}),(\mathrm{d})$ ) is more comparable to that obtained by Miranda et al. (2009) than that seen when the BLH is used (figure 6(a),(b)), despite the resolution refinement between protected by copyright. All rights reserved shifted from JJA to SON (not shown), although the difference between these two seasons is small, as already noted.

Global plots equivalent to figure 6 (not shown) additionally indicate that one of the few locations where drag is reduced by shear is over the Andes, suggesting that the shear in this region is predominantly linear (Teixeira et al. 2004). The drag enhancement over the edge of Antarctica that is displayed in figure 6 suggests some degree of robustness of the drag behaviour both to changes in the height in the atmosphere at which the shear terms are evaluated (as the two panels are qualitatively similar) and to orography anisotropy (since the present calculation uses an axisymmetry assumption, but the results resemble those of Miranda et al. (2009)). This is discussed further next.

\subsubsection{Elliptical mountains}

We now consider orography having an elliptical horizontal crosssection, as used to approximate the subgrid-scale orography in drag parametrizations of leading GCMs (Lott and Miller 1997). The parameters that concern us here are $\gamma$, the aspect ratio of the elliptical horizontal cross-section of the mountain, and $\phi$, its orientation angle. Both quantities, which enter in (2)(3) and in the coordinate transformation that must be applied before these two equations (expressed in a coordinate system aligned with the main axes of the ellipse (Teixeira and Miranda 2006)) can be used, are shown in figure 7 for Antarctica. Both are available directly from the ERA-Interim reanalysis database. Actually the absolute value of $\phi$ is displayed is figure 7(b) because the interpretation that follows only depends on whether $\phi$ corresponds to zonal or meridional orography. In ERA-Interim, while $\phi=0^{\circ}$ denotes meridional orography orientation, both $\phi=$ $\pm 90^{\circ}$ denote zonal orientation. In figure $7, \gamma$ varies between 0 and 1 (corresponding, respectively, to a $2 \mathrm{D}$ and an axisymmetric mountain). The coordinate transformation mentioned above may be expressed as (see Teixeira and Miranda 2006)

$$
\begin{aligned}
U & =\hat{U} \cos \phi+\hat{V} \sin \phi, \\
V & =\hat{V} \cos \phi-\hat{U} \sin \phi,
\end{aligned}
$$

where $U$ and $V$, and $\hat{U}$ and $\hat{V}$ denote, respectively, the wind components aligned with the minor and major axes of the ellipse (i.e. those in (2)-(3)), and aligned in the zonal and meridional directions (i.e. those provided by reanalysis). Figure 7 shows that most of the orography of Antarctica, in particular that bordering on the Antarctic ocean (except perhaps in parts of the Antarctic Peninsula) is quite anisotropic. It is also clear that the the orientation of the orography towards most of the edge of Antarctica is predominantly zonal, which is not surprising, since the edge of Antarctica itself is in most places roughly zonal. The consequences of these features for the drag behaviour will be discussed next.

Figure 8(a),(c) shows the drag enhancement (as in figure 6) for elliptical orography, but limited to the JJA season, since this is roughly when this enhancement is maximized. Figure 8(b),(d) shows the corresponding dimensional GWD stress enhancement due to shear (in Pa), i.e. the GWD stress (drag per unit area) in the absence of shear effects multiplied by the shear correction. This latter result is used to illustrate the true magnitude of the enhancement effect that can be expected in a parametrization context, where the GWD stress is the quantity that is directly parametrized. Drag enhancement is seen throughout the year in the Antarctic region, but it is more modest than for axisymmetric orography. The maximum enhancement values (mostly between 1.2 and 1.3) are somewhat spotty spatially and occur during JJA when the BLH is used as the reference height, and during SON when the SDH is used instead (not shown), but the difference 


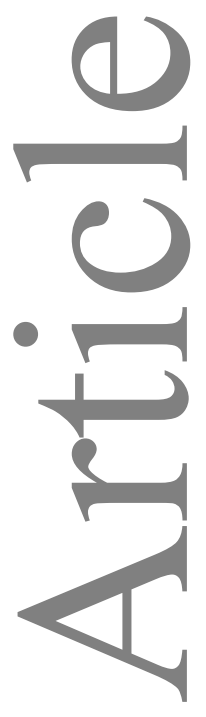

(c)

(a)

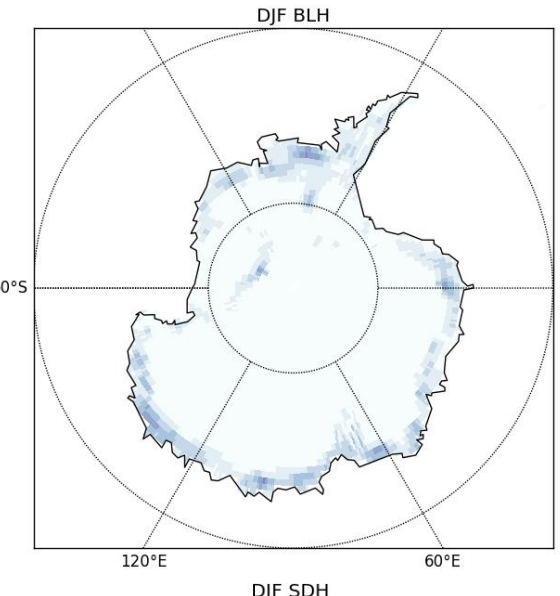

DJF SDH

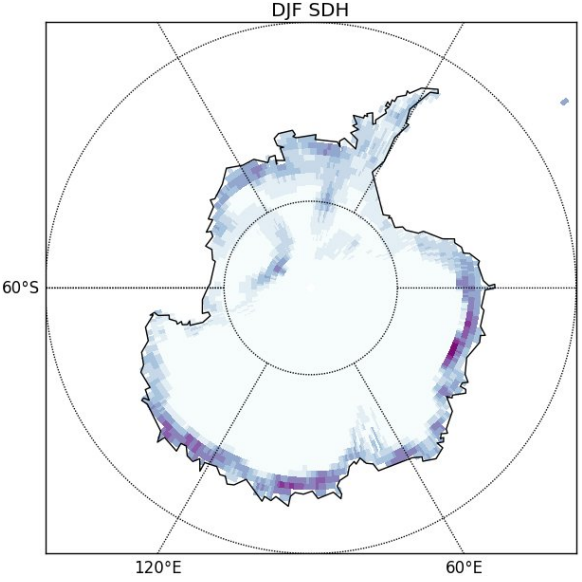

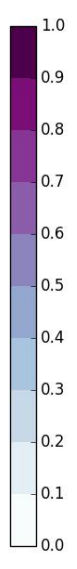

(b)
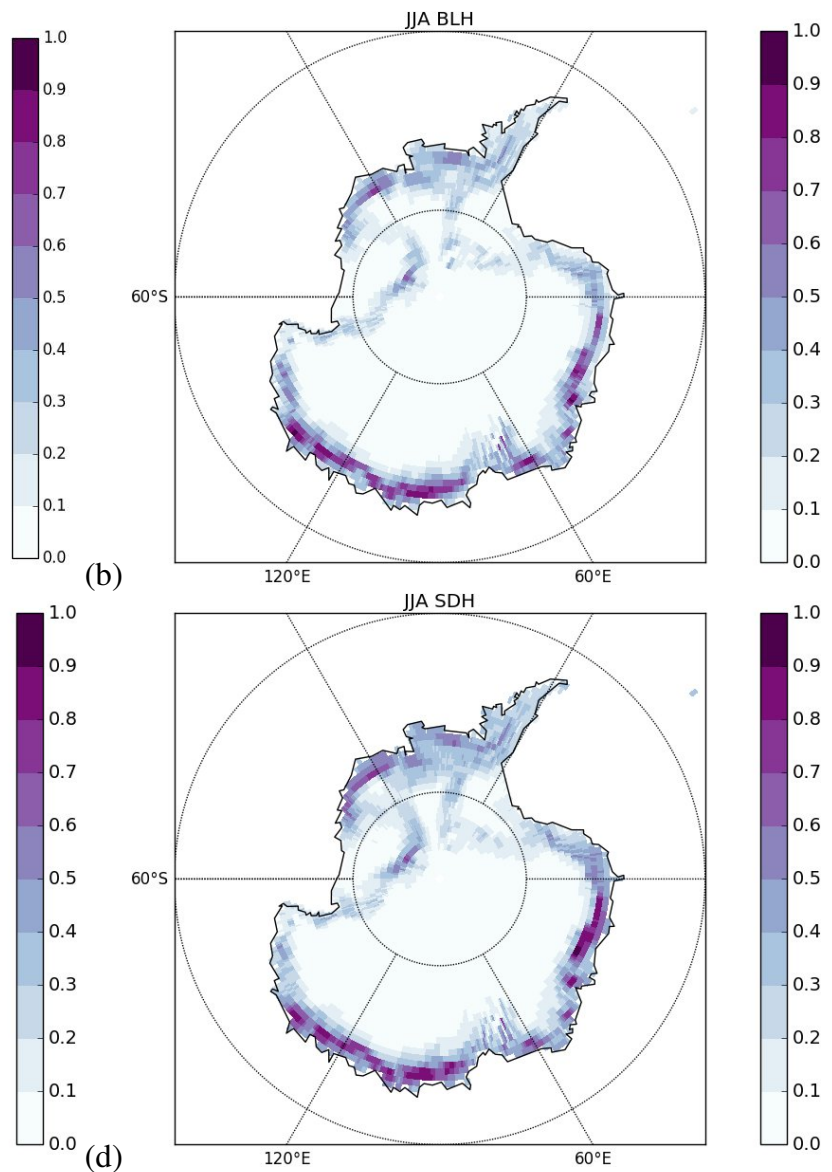

Figure 5. Fraction of the time at which $\mathrm{Ri}^{-1}>1 / 3$ over Antarctica during the decade 2006-2015 for (a) DJF at the BLH, (b) JJA at the BLH, (c) DJF at the SDH, (d) $\mathrm{JA}$ at the SDH.

()

JJA is small, so we opted for displaying values for JJA (with maxima mostly above 1.4) to keep a uniform approach. The enhancement of the GWD stress shown in Figure 8(b),(d) follows the dimensionless drag enhancement qualitatively, having a larger magnitude, with maxima of between 0.5 and $1 \mathrm{~Pa}$, when the SDH is used, and being mostly between 0.2 and $0.5 \mathrm{~Pa}$ when the BLH is used instead. Both of these values are of the same order of magnitude as the maximum GWD stress perturbations due to shear found by Miranda et al. (2009) for July 2001 (see their figure

6), but the value using the SDH is numerically closer. These values clearly practically relevant, since the total GWD stress itself is typically of a few Pa (Miranda et al. 1999).

It is interesting that the drag enhancement seen in figure (a),(c) for both reference heights is not as strong as that seen for axisymmetric mountains in figure 6 . This must be due to the orientation of the wind field with respect to the mountains. It is well-known that the drag magnitude is larger when the wind is perpendicular to an anisotropic mountain than when it is parallel to it (Phillips 1984). Teixeira and Miranda (2006) further showed that the drag corrections due to shear are larger when the shear is directed across an anisotropic mountain than along it.

Figure 9 shows a histogram of the angle between the shear vector, $\left(U^{\prime}, V^{\prime}\right)$ over Antarctica and the minor axis of the elliptical mountains used to represent the orography in each reanalysis grid box. Similarly to $|\phi|$, this angle only takes values between $0^{\circ}$ and $90^{\circ}$, since any other angle would be redundant in terms of relative direction, and can always be expressed as equivalent to an angle within this interval. We can see that this angle peaks near $90^{\circ}$, and has a minimum at $0^{\circ}$, which means that the shear is predominantly aligned along the mountains (slightly less so when evaluated at the SDH - This article isp pratected dy copyrteht A.ll rights reserved the drag corrections have a smaller magnitude in this situation, which explains why in figure 8(a),(c) the drag is less enhanced at the edge of Antarctica than in figure 6. Taking into account that the thermal gradients at the edge of Antarctica are likely to be mostly meridional, given that the temperature decreases strongly inland towards the South, by the thermal wind relation the wind should have predominantly zonal shear. Conjugated with the predominantly zonal orientation of the orography at the edge of Antarctica, this probably produces the shear alignment with the long axis of the orography that can be seen in figure 9 .

To further characterize the drag corrections for the elliptical orography, figure 10 shows histograms of the drag enhancement over Antarctica, discriminated by season, for the decade 20062015, for shear corrections calculated at the BLH (figure 10(a),(b)) and at the SDH (figure 10(c),(d)). Inspection of the histograms for both calculation heights reveals that the most common values of drag enhancement are between 1.0 and 1.1. The drag can be reduced due to shear, but invariably by a factor not smaller than 0.9. The histograms demonstrate, on the one hand, that the drag is predominantly enhanced by shear effects, and on the other hand, that this enhancement is typically small, especially in the case where the BLH is used. Significant drag enhancement, in the range 1.3-1.5, is possible when estimating shear terms at the SDH,but it is still very unlikely and infrequent. This is consistent with the maps of figure 8 . Another aspect to note is that the seasonal peak in intensity of the drag enhancement shifts from JJA when the BLH is used, to SON when the SDH is used instead (not shown), which agrees with the results discussed above in connection with figure 8. However, again, the difference between SON and JJA (only the latter of which is shown in figure 10(d)) is small. 

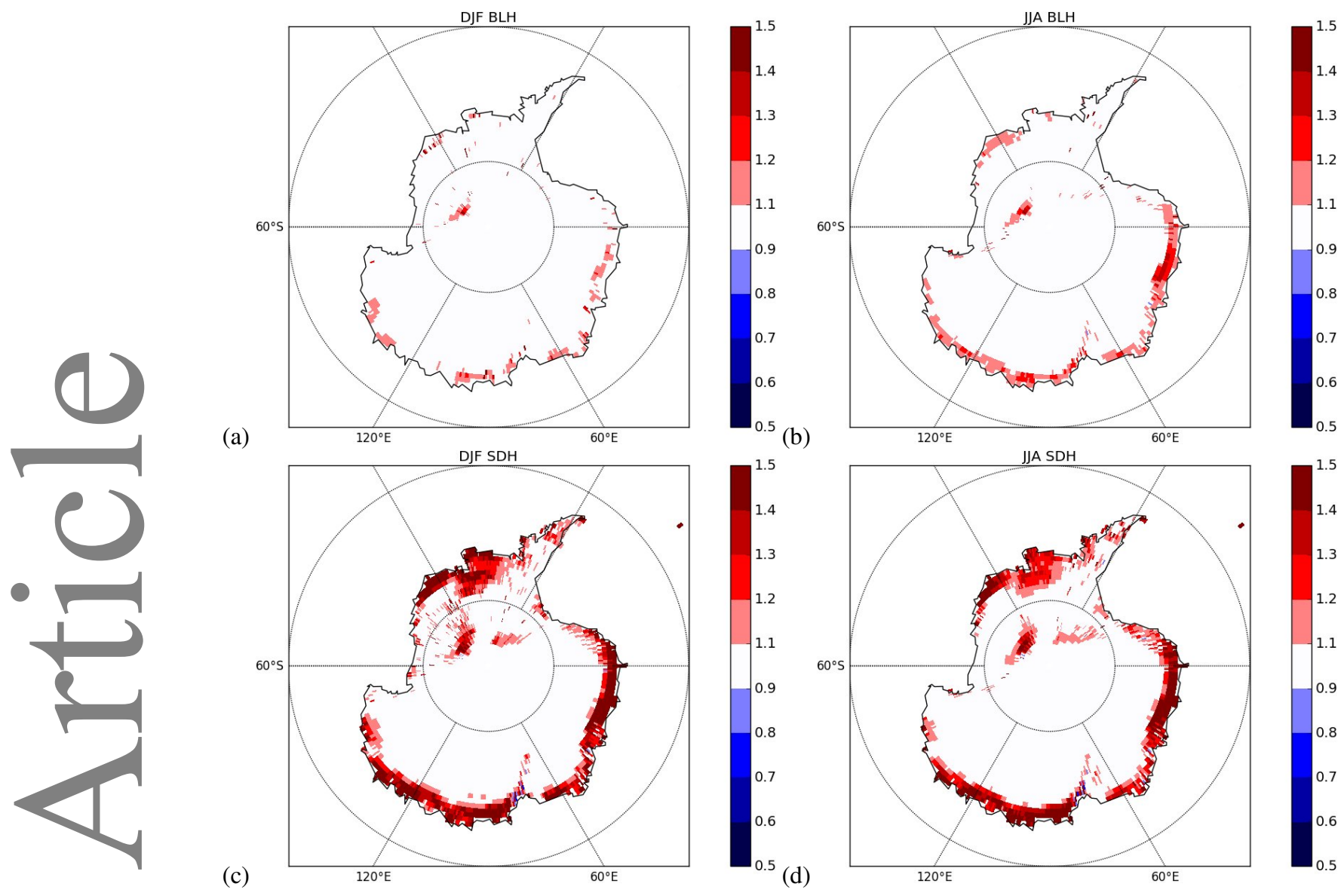

(b)

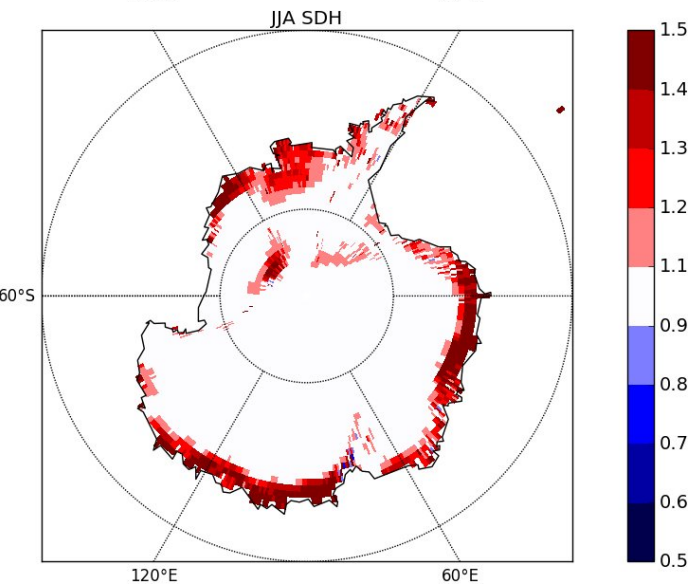

Figure 6. Drag enhancement over Antarctica for the decade 2006-2015, using an axisymmetric subgrid-scale orography approximation for (a) DJF at the BLH, (b) JJA at the BLH, (c) DJF at the SDH, (d) JJA at the SDH.
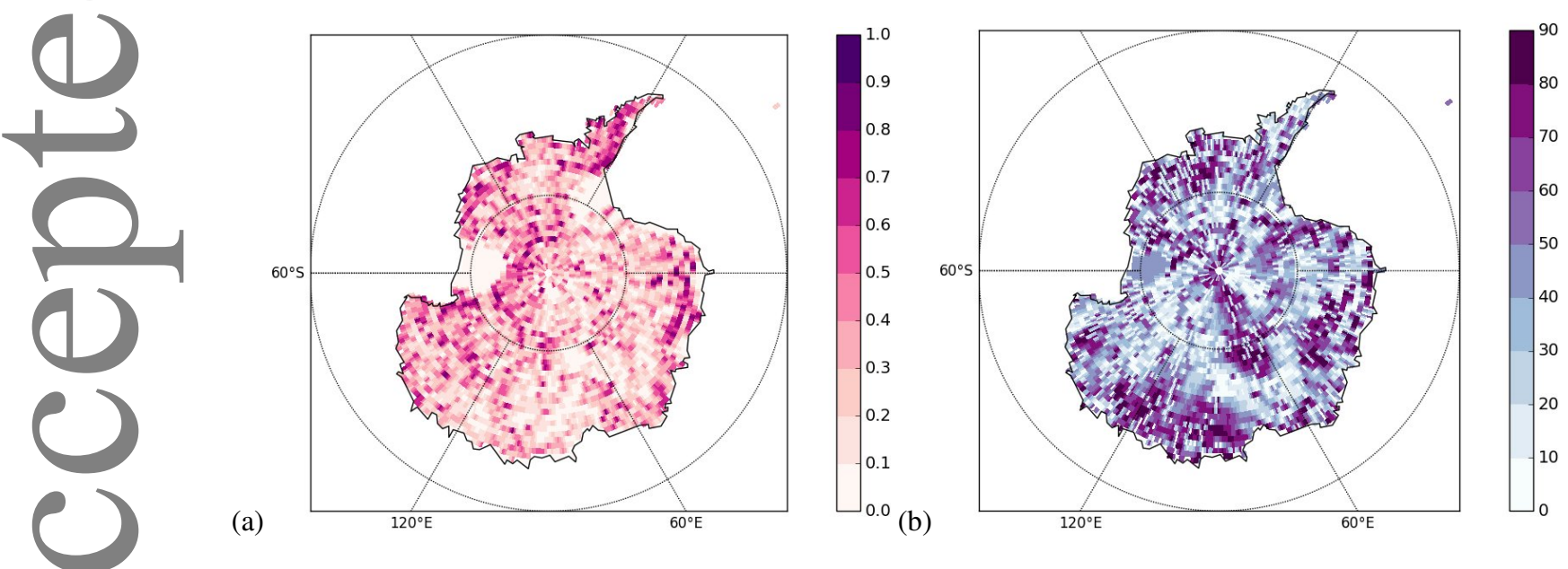

Figure 7. (a) Orography aspect ratio $(\gamma)$, assuming that the subgrid-scale orography within each grid box can be represented by a mountain with an elliptical horizontal cross-section, and (b) absolute value of orientation angle $(|\phi|)$ of that elliptical mountain.

\subsection{The relative impact of wind profile shear and curvature}

To further assess the relative impacts of terms involving first and second wind derivatives (the latter often associated with directional shear for reasons explained above) upon the drag corrections, we split equations (2)-(3) appropriately so as to separate those terms. We then compare the magnitudes of the two totals corresponding to the sum of all terms of each type, to evaluate the fraction of times at which the contributions of each type of derivatives dominate. The results of this analysis are shown in figure 11, which displays the fraction of times that terms involving second derivatives dominate over those involving only

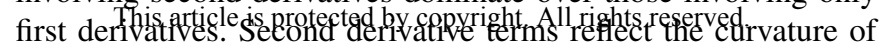

the wind profile, and typically have an enhancing effect on the drag. As explained previously, this is because the fact that the wind speed is bounded implies that, on average, a given wind velocity component and its curvature have opposite signs. Now, the terms involving $U U^{\prime \prime}$ and $V V^{\prime \prime}$ are preceded by a minus sign that makes them typically positive (see (2)-(3)). First derivative terms representing a linear wind variation, on the other hand, almost always give a negative correction to the drag because they appear squared and with a minus sign (with the exception of the crossterms involving $U^{\prime} V^{\prime}$ ). Thus, we expect that second derivatives will be dominant in areas where drag enhancement is detected (cf. figure 8). From a physical standpoint, it can be shown that GWD is proportional to the real part of the vertical wave number 


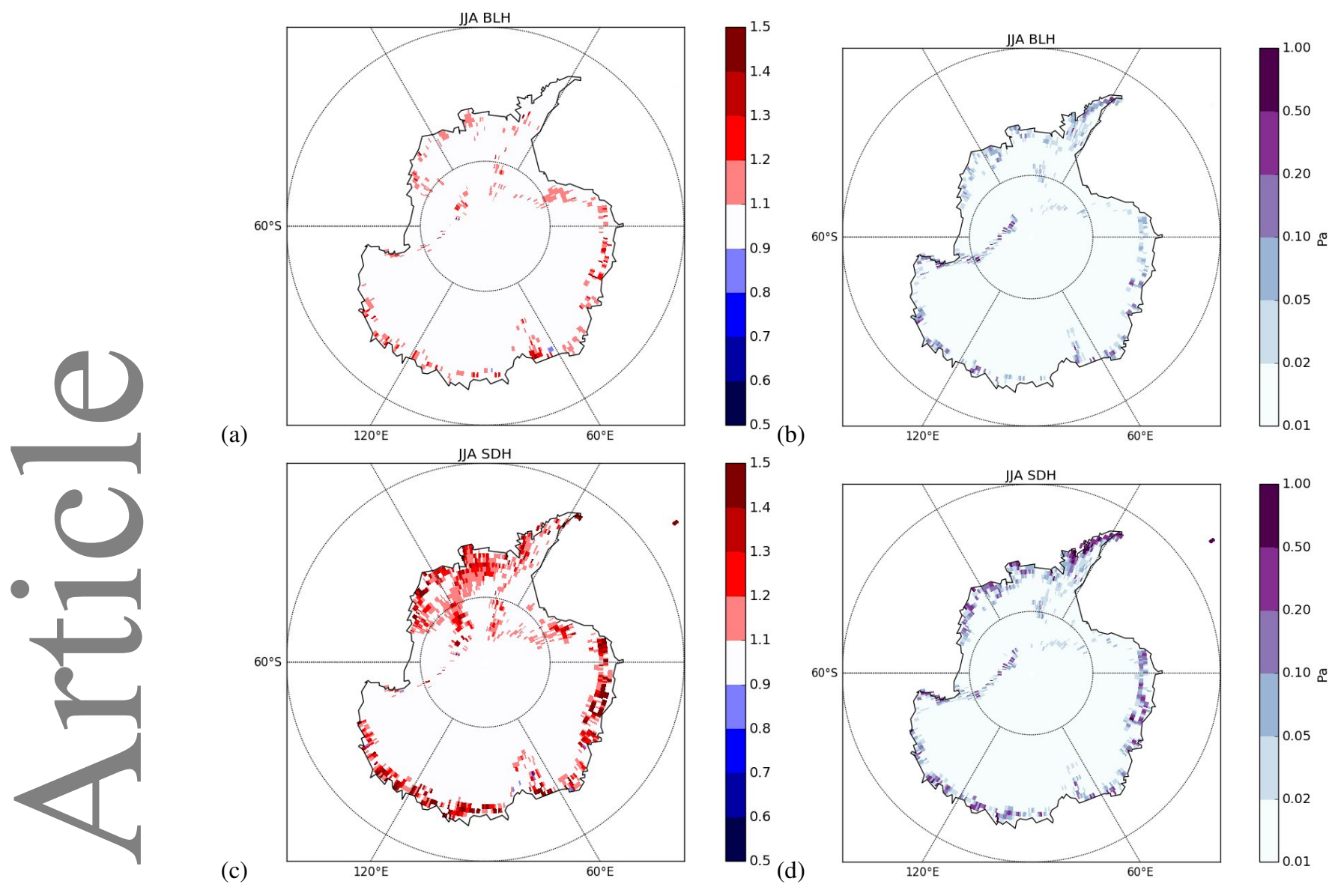

Figure 8. Drag enhancement by shear ((a),(c)) and absolute enhancement of the GWD stress (in Pa) ((b),(d)) over Antarctica, with shear corrections computed at the BLH (a),(b)) and at the SDH ((c),(d)), during JJA for the decade 2006-2015, using subgrid-scale orography with an elliptical horizontal cross-section.
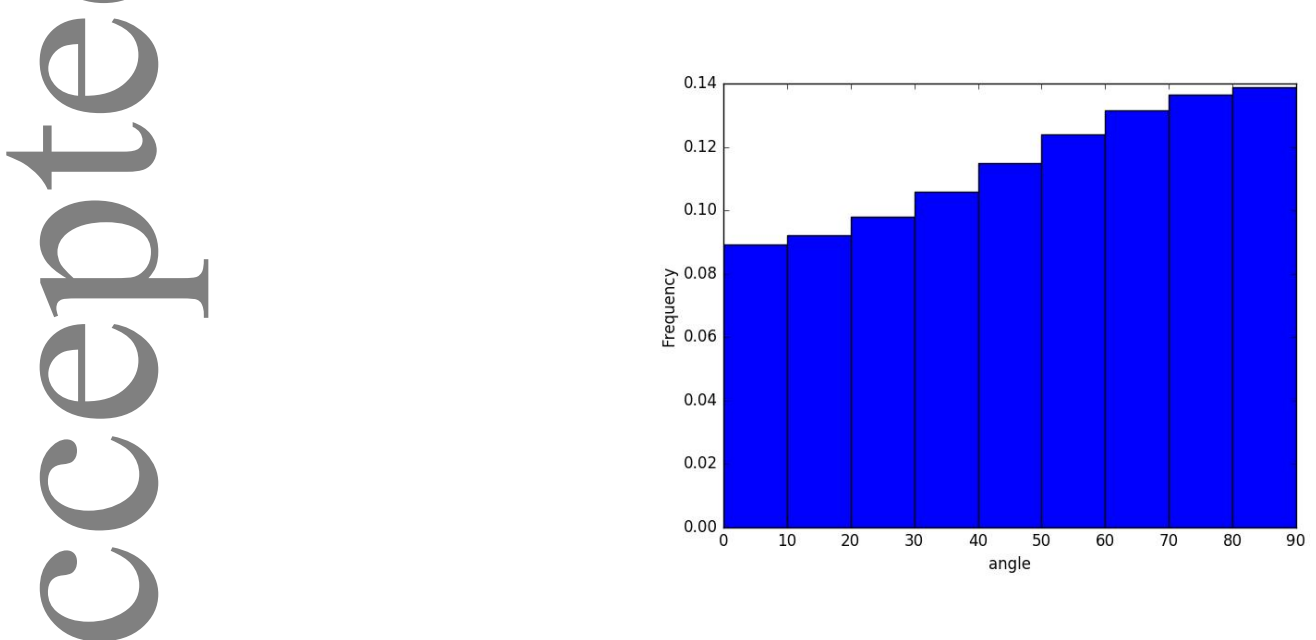

Figure 9. Histogram of the orientation of the shear vector relative to the orography over Antarctica, during the decade $2006-2015$ for JJA at the BLH. $0^{\circ}$ and $90^{\circ}$ correspond to flow across and along a mountain ridge, respectively.

of the gravity waves and that negative curvature (which is seen in wind turning) increases this wavenumber (Teixeira and Miranda 2006). In order to lead to drag enhancement, the curvature terms in the drag correction formulae need not only to balance but also to dominate over linear terms. Miranda et al. (2009) showed theoretically that, on average, curvature terms indeed tend to dominate (see their Appendix). It is interesting that the peak in the dominance of wind profile curvature effects occurs during SON using both reference heights: BLH and SDH (see figure 11). This does not, however, necessarily correspond to a maximum of drag enhancement (using the BLH the maximum enhancement occurs in JJA Thiss article is protected bot copycight, All rights reserved if terms involving second derivatives are the largest ones they may not necessarily be large enough to produce the maximum drag values.

\section{Conclusions}

This study has shown that wind profile shear is likely to affect GWD all year round over the globe, with the distribution and intensity of this effect (measured to a first approximation using the Richardson number) varying on a seasonal timescale. Polar areas in winter are characterized by low values of Ri, which seem to be the most reliable. One of the regions affected most by shear appears to be the edge of Antarctica, confirming the findings of Miranda et al. (2009). However, the present study goes beyond 
those previous calculations by testing the sensitivity of the shear corrections to the drag to two choices of height where they are evaluated, defined to avoid near-surface boundary layer effects of minor relevance to mountain wave dynamics. One of these heights corresponds to the first reanalysis levels above the diagnosed boundary layer top, BLH. The other height, SDH, corresponds to 1.5 standard deviation of the subgrid-scale orography elevation within the model grid box above the ground. This latter choice is formally similar to that of Miranda et al. (2009), but differs in value because of the different resolutions between ERAInterim reanalysis (used here) and ERA-40 reanalysis (adopted by Miranda et al. (2009)).

Results both for $\mathrm{Ri}^{-1}$ (an overall measure of the importance of shear) and for the actual shear corrections to the drag suggest that those corrections are qualitatively robust to changes in the height at which the vertical derivatives of the wind velocity are approximated. Namely, their geographic distribution, seasonal cycle and average sign are essentially the same. However, quantitatively, the drag enhancement diagnosed for Antarctica by evaluating the shear corrections at the BLH and at the SDH height do produce different results. Specifically, the shear is considerably more enhanced using the SDH than using the BLH (by a factor of 2 or more), primarily because the SDH is typically lower than the BLH, and shear tends to concentrate near the ground. These results extend to the values of the (dimensional) correction to the GWD stress due to shear, which reaches maxima with a practically relevant magnitude that are larger (and similar to those of Miranda et lal. (2009)) when SDH is adopted, and somewhat smaller when BLH is adopted instead. A more detailed examination of the relative magnitudes of terms in the drag expressions that contain first and second derivatives indicates that terms containing second derivatives (i.e. curvature terms) are dominant in a high fraction the region, over a large fraction of the time. We believe that use of the BLH is more justified physically than that of the SDH, particularly in regions of the world where deep and statically unstable or neutral boundary layers occur. In these locations, the SDH may well lie within the boundary layer and thus estimating shear effects at that level could lead to unphysical results.

The sensitivity of the shear-induced drag corrections to orography anisotropy was also tested by computing those corrections for the "realistic" elliptical orography, with parameters obtained from reanalysis, and for an axisymmetric orography approximation, where such parameters become unnecessary. The latter approach considerably overestimated shear effects, because the shear was directed more often along the orography than across it at the edge of Antarctica. The results are, nevertheless, not as sensitive to orography anisotropy as they are to the reference height, suggesting that the nature of the shear matters more for drag enhancement than the orography anisotropy. The direction of the drag vector relative to the orography (not addressed here) is, course, also important, and worthy of investigation. However, this is likely to be quite a noisy field, as suggested by the map of the angle of the orography (figure 7(b)).

The main conclusions of this study (answering the questions formulated in the Introduction) can be summarized as follows:

- The shear-induced drag correction is qualitatively robust to changes in the height at which the vertical derivatives are estimated.

- The drag is considerably more enhanced when the SDH is used as the reference height rather than the BLH.

- Use of an axisymmetric orography assumption results in considerable overestimation of the shear effects.

- Terms containing second derivatives dominate the drag correction for a large fraction of time during all seasons, indicating that directional wind shear dominates the drag thris articiei isprotected by copyright. All rights reserved.
The aim here was to test the sensitivity of the drag to two choices of reference height used for evaluating shear effects. An objective determination of the optimal height for this purpose (if it exists) is non-trivial, and a topic for further study.

\section{Acknowledgement}

We thank Alice Gilbert (née Baker), who provided preliminary versions of the Python codes used to process the reanalysis data. HVT acknowledges the financial support of NERC under PhD studentship 1641628.

\section{References}

Bender CM, Orszag SA. 1999. Advanced mathematical methods for scientists and engineers. Springer.

Gregory D, Shutts GJ, Mitchell JR. 1998. A new gravity-wave-drag scheme incorporating anisotropic orography and low-level wave breaking: Impact upon the climate of the uk meteorological office unified model. Quart. J. Roy. Meteor. Soc. 124: 463-493.

Kim YJ, Eckermann SD, Chun HY. 2003. An overview of the past, present and future of gravity-wave drag parametrization for numerical climate and weather-prediction models - survey article. Atmosphere-Ocean 41: 65-98.

Lott F, Miller MJ. 1997. A new subgrid-scale orographic drag parametrization: Its formulation and testing. Quart. J. Roy. Meteor. Soc. 123: 101-127.

McFarlane NA. 1987. The effect of orographically excited gravity wave drag on the general circulation of the lower stratosphere and troposphere. $J$. Atmos. Sci. 44: 1775-1800.

Miranda PMA, Ferreira JJ, Thorpe AJ. 1999. Gravity-wave drag produced by madeira. Quart. J. Roy. Meteor. Soc. 125: 1341-1357.

Miranda PMA, Martins JPA, Teixeira MAC. 2009. Assessing wind profile effects on the global atmospheric torque. Quart. J. Roy. Meteor. Soc. 135 : 807-814.

Palmer TN, Shutts GJ, Swinbank R. 1986. Alleviation of a systematic westerly bias in general circulation and numerical weather prediction models through an orographic gravity wave drag parametrization. Quart. J. Roy. Meteor. Soc. 112: 1001-1039.

Phillips DS. 1984. Analytical surface pressure and drag for linear hydrostatic flow over three-dimensional elliptical mountains. J. Atmos. Sci. 41: 10731084.

Sodemann H, Masson-Delmotte V, Schwierz C, Vinther BM, Wernli H. 2008. Interannual variability of greenland winter precipitation sources: 2 . effects of north atlantic oscillation variability on stable isotopes in precipitation. $J$. Geosphys. Res. 113: D12 111.

Teixeira MAC, Miranda PMA. 2004. The effect of wind shear and curvature on gravity wave drag produced by a ridge. J. Atmos. Sci. 61: 2638-2643.

Teixeira MAC, Miranda PMA. 2006. A linear model of gravity wave drag for hydrostatic sheared flow over elliptical mountains. Quart. J. Roy. Meteor. Soc. 132: 2439-2458.

Teixeira MAC, Miranda PMA, Valente MA. 2004. An analytical model of mountain wave drag for wind profiles with shear and curvature. J. Atmos. Sci. 61: 1040-1054.

Wallace JM, Tibaldi S, Simmons AJ. 1983. Reduction of systematic forecast errors in the ecmwf model through the introduction of an envelope orography. Quart. J. Roy. Meteor. Soc. 109: 683-717.

Watanabe S, Sato K, Takahashi M. 2006. A general circulation model study of the orographic gravity waves over antarctica excited by katabatic winds. $J$. of Geophys. Res. 111: D18 104.

Zadra A, Roche M, Laroche S, Charron M. 2003. The subgrid-scale orographic blocking parametrization of the gem model. Atmosphere-Ocean 41: 155170 . 